\title{
On Fairness in the Network Air Traffic Flow Management with Rerouting
}

\author{
Sadeque Hamdan ${ }^{* \#}$, Ali Cheaitou ${ }^{* 1}$, Oualid Jouini" ${ }^{\#}$ Zied Jemai $^{3}$, Imad Alsyouf*1, Maamar Bettayeb ${ }^{2}$ \\ *Sustainable Engineering Asset Management (SEAM) Research Group, ${ }^{1}$ Industrial Engineering and Engineering \\ Management Dept., ${ }^{2}$ Electrical and Computer Engineering Dept. University of Sharjah, Sharjah, United Arab Emirates. \\ "Laboratoire Genie Industriel, CentraleSupélec, Université Paris-Saclay, 91192 Gif-sur-Yvette, France. \\ ${ }^{3}$ OASIS - ENIT, University of Tunis Elmanar, Tunisia. \\ e-mail: sadeque.hamdan@supelec.fr; acheaitou@sharjah.ac.ae; oualid.jouini@ecp.fr; zied.jemai@ecp.fr; \\ ialsyouf@sharjah.ac.ae; maamar@sharjah.ac.ae
}

Abstract - This paper presents a fairness model for the network air traffic flow management with rerouting under deterministic capacity. In addition to ground delay and air delay, the model provides control on the aircraft speed through the control of the time spent in each sector, and the ability to reroute the aircraft to avoid or reduce delays. The model covers two aspects of fairness, which are delay distribution and flights reversal. The model outperforms existing works in terms of the number of delayed flights per time period as well as the total and maximum delay.

Keywords-air traffic flow management; reversal; rerouting; fairness; ground delay; air delay.

\section{INTRODUCTION}

Major airports are facing serious congestion, where airports' runways and airspace capacities are the major source of this congestion [1]. One of the solutions to better manage the congestion and the huge amount of delays is the ground delay programs which aim to delay the departure of flights in order to avoid any airborne delay and to give more priority for arriving flights and for the en-route flights to use the airspace. The justification for this action is that the air delay cost is much higher than the one for ground delay due to the fuel cost, safety issues, etc. [2]. However, such ground delay programs do not necessarily consider the entire network elements. In the air traffic flow management (ATFM) problem not only airport capacities but also sector capacities (capacitated airspace) are considered in a strategic processes to achieve less congestion, more safety and efficiency in traffic movement [3]. ATFM decisions affect critically the involved aviation stakeholders, where there is a need to exchange 
information and to have a significantly enhanced decision-making process between the different partners to fit into the collaborative decision-making philosophy. ATFM decisions can be related to air delay, ground delay or to speed controls.

The consideration of rerouting decisions, i.e. changing the flight path to avoid congested sectors in the airspace, can be another option to reduce the total delay, which leads to ATFMRP (air traffic flow management with rerouting problem). Decision makers need in this situation to be fair when making the different decisions, for instance when assigning delays to the different airlines. The purpose of this paper is the optimization of the air traffic flow management with rerouting possibilities (FairnessATFMRP), while accounting for fairness. This extends the work of [3]. We find that the proposed model enhances flights scheduling and reduces the number of delayed flights and the total amount of delay.

The organization of the paper is as follows: Section II presents the work done in this field and the contribution of this paper. Section III describes the problem and Section IV formulates the mathematical model. Section V illustrates the model functionalities and provides some insights. Section VI concludes the paper.

\section{LITERATURE REVIEW}

One possible classification of the mathematical optimization models in the field of air traffic management to balance the demand and the capacity is: the single airport ground delay problem, the multiple airport ground delay problem, the air traffic flow management (ATFM) problem and the air traffic flow management with re-routing (ATFMRP) [2], [4].

In the single airport ground delay models, the problem formulation considers only one airport and aims to balance the demand and the capacity by providing ground delays to the aircrafts [4] see among others ([5], [6]). While in the multiple airport ground delay models, a set of airports is considered which allows the consideration of connecting aircrafts but this lacks the ability to provide decisions during the flight such as speed control, and does not consider the airspace capacity ([4], [7]).

The ATFM problem extends the multiple airport ground delay problem to consider the airspace, which provides more control and decisions on the flights ([8]-[12]). The consideration of changing the path of a flight leads to the ATFMRP, which is a problem that has been addressed in the literature. For instance, Bertsimas and Patterson [13] studied the deterministic ATFM problem under changing weather environment with consideration of rerouting. After that, Bertsimas et al. [14] developed an integer programming deterministic model to solve large-scale instances for flight-by-flight ATFMRP. The proposed model 
controls each flight throughout its entire duration while considering fairness in ground-delay and air-delay, rerouting, and speed control through the adjustment of the time spent in each sector.

Moreover, Mukherjee and Hansen [1] developed three stochastic ATFM models that can use probabilistic information to efficiently manage air traffic flow and support ground holding and rerouting decisions. The first model is for static ground holding without rerouting. The second model considers static ground holding and static rerouting. The third model represents static ground holding and dynamic rerouting decisions. The model considers a macroscopic case where an aggregated number of flights planned to arrive at various resources (sectors or airports) is the decision variable rather than a microscopic case of flight-by-flight control.

Inspired from Bertsimas and Patterson [4] and Mukherjee and Hansen [16], Agustín et al. [2] proposed a deterministic multiobjective mixed integer binary programming model using arc formulation for flight-by-flight ATFM problem. They considered rerouting, flight cancelation, continuing flights and fairness in ground and air delay assignment that was introduced by Lulli and Odoni [17]. Agustín et al. [18] extended their deterministic version of the model in [2] to a stochastic model that accounts for uncertainty in airport arrival and departure capacities' and sectors capacities'.

In the literature, fairness in the network has been addressed under two aspects. The first aspect lies in the fairness in the distribution of the delay among the flights, so instead of delaying a flight by two units of time, the delay is split into one unit of time for two different flights. This concept was introduced in Lulli and Odoni [17] by using slightly super linear cost coefficients for the delay objective function. The second aspect of fairness is achieved through the control of the reversal between the flights which was introduced in [3].

This paper contributes to the literature by extending the work of [3] and [2] to allow for rerouting in the flight path in a fair manner, where Bertsimas and Gupta [3], did not consider the rerouting aspect in their model and Agustín et al. [2] did not consider fairness aspects between flights. The rerouting option will provide more options and flexibility in scheduling the flights in a more fair way instead of using fixed routes. This option will also help in reducing the delays in the network as well.

\section{FAIRNESS-ATFMRP PROBLEM DESCRIPTION}

The model proposed in this paper assumes that a given set of scheduled flights is expected to take off from and to land in a network of airports with another set containing a pre-defined route for each flight. The routes pass through a set of sectors in the 
airspace system during a set of discrete time-periods of 15-minutes each forming the planning horizon. Each sector has its own capacity that depends on the sector size, controller workload, weather and other factors. Each airport has its own departing and arriving capacity. The problem aims to balance the demand of flights and the capacity of the different resources, i.e. sectors and airports. This goal can be achieved by applying a set of decisions and controls such as speed control on some sectors, ground delay, air delay and rerouting. In this work, rerouting is considered as a group of pre-defined paths, and the model selects one of these paths from the available group and adds a penalty cost if the selected path differs from the planned one. This approach of defining a limited group of potential routes for each flight can be motivated by practical considerations [1]. While aiming to balance the demand and the capacity of the airspace system, fairness between flights should be taken into consideration. Flight fairness is considered in distributing the delay among the flights and in the control of the reversal between the flights.

\section{MATHEMATiCAL Model}

A. Sets

- $\quad \mathcal{F}$ : Set of flights, $f=1, \ldots, \mathrm{F}$.

- $\mathcal{T}$ : Set of discrete finite time periods of 15 min duration each, $t=1, \ldots, T$.

- $\mathcal{K}:$ Set of airports in the network $k=1, \ldots, K$.

- $\quad Z_{f}:$ Set of alternative routes belonging to flight $f, z_{f}=1, \ldots, Z_{f}$.

- $p_{f}^{z_{f}}$ : Set of sectors that flight $f$ is supposed to follow in alternative $z_{f}, j \in p_{f}^{z_{f}}$.

- $\mathcal{R}$ : Set of reversal flights, each element in the set contains the following four values $\left(f, z_{f}, f^{\prime}, z_{f^{\prime}}\right)$.

- $\quad \mathcal{C}$ : Set of pair of flights that are continuing $\left(f, f^{\prime}\right)$.

- $\quad T_{j}^{f, z_{f}}$ : List of possible flight time periods for flight $f$ in resource $j \in p_{f}^{z_{f}} \cup \mathcal{K}$ (i.e. airport or sector) using alternative $z_{f}$. 


\section{B. Parameters}

- $\mathrm{C}_{\text {air }}, \mathrm{C}_{\text {ground }}$ : Penalty cost for delaying a flight in the air or for delaying the departure of a flight for one time unit respectively.

- $\quad c_{\text {arr }}^{f}(t), c_{\text {dep }}^{f}(t)$ : Functions that calculate the total air delay and ground delay for flight $f$ based on the delay in the flight arrival and departure.

- $\operatorname{orign}_{f}$ : Departure airport of flight $f$.

- $\quad \operatorname{dest}_{f}$ : The main destination airport of flight $f$.

- $p_{f}^{z_{f}}(j+1)$ : Subsequent sector of the $j^{\text {th }}$ sector for flight $f$ in the alternative route $z_{f}$.

- $p_{f}^{z_{f}}(j-1)$ : Preceding sector of the $j^{\text {th }}$ sector for flight $f$ in the alternative route $z_{f}$.

- $D_{k}(t)$ : departure capacity of the runway in airport $k$ at time $t$.

- $A_{k}(t)$ : arrival capacity of the runway in airport $k$ at time $t$.

- $S_{j}(t)$ : Capacity of sector $j$ in the network at time $t$

- $\quad a_{f}^{j, z_{f}}$ : Arrival time for flight $f$ in resource $j$ using $z_{f}$ route.

- $\quad z_{f}^{S}$ : the scheduled route for flight $f$

- $\quad d_{f}^{k}$ : scheduled departure time for flight $f$ from airport $\mathrm{k}$.

- $s_{f}$ : The minimum turnaround time needed for flight $f$ to take-off after the arrival of flight $f^{\prime}$ in the case of continuing flights for each $\left(f, f^{\prime}\right) \in \mathcal{C}$.

- $\quad \overline{\bar{T}}_{j}^{f, z_{f}}, \underline{T}_{j}^{f, z_{f}}$ : the first and the last time period in the set $T_{j}^{f, z_{f}}$ : the earliest possible time and the latest possible time for flight $f$ to enter resource $j$ using altnerative $z_{f}$ 
- $\quad C_{R \text { airport }}^{f}, C_{R}^{f}$ sector : the costs of reversal at arrival airport and sector for flight $f$ respectively.

- $C_{\text {reroute }}^{f, z_{f}}$ : The cost of choosing the alternative path $z_{f}$ for flight $f$. For the scheduled path $C_{\text {reroute }}^{f, z_{f}^{s}}=0$.

\section{Decision Variables}

- $\quad w_{j, t}^{f, z_{f}}$ : A binary variable equals to one if flight $f$ arrives to resource $j$ by time $t$ using path alternative $z_{f}$. Otherwise it is equal to zero. If $w_{j, t}^{f, z_{f}}=1$ at any time period $\mathrm{t}$, then it will be equals to one for all the later periods.

- $R_{j, f, f^{\prime}}^{z_{f}, z_{f^{\prime}}}$ : A binary variable that is equal to one if a reversal occurs between flight $f$ from route $z_{f}$ and flight $f^{\prime}$ from route $z_{f^{\prime}}$ at resource $j$. It is equal to zero otherwise.

\section{Mathematical Model}

The objective function and constraints for the mathematical model are presented as follows:

$$
\begin{aligned}
& \min C=\sum_{f \in \mathcal{F}} \sum_{z_{f} \in z_{f}}\left(\sum_{\substack{f, z_{f} \\
t \in \text { dest }_{f}}} c_{\text {arr }}^{f}(t)\left(w_{\text {dest }_{f}, t}^{f, z_{f}}-w_{\text {dest }_{f}, t-1}^{f, z_{f}}\right)+\sum_{\substack{f, z_{f} \\
t \in T_{\text {orign }}}} c_{\text {dep }_{f}}^{f}(t)\left(w_{\text {orgin }_{f}, t}^{f, z_{f}}-w_{\text {orgin }_{f}, t-1}^{f, z_{f}}\right)\right) \\
& +\sum_{f \in \mathcal{F}} \sum_{z_{f} \in \mathcal{Z}_{f} /\left\{z_{f}^{S}\right\}} C_{\text {reroute }}^{f, z_{f}} \times\left(w_{\text {orign }_{f}, T}^{f, z_{f}}\right)+\sum_{f \in \mathcal{F}} \sum_{k=\text { dest }_{f} k \in \mathcal{K},\left(f, z_{f}, f^{\prime}, z_{f^{\prime}}\right) \in \mathcal{R},} C_{R_{\text {airport }}}^{f} \times R_{k, f, f^{\prime}}^{z_{f, z_{f}}} \\
& +\sum_{f \in \mathcal{F}} \sum_{j \in p_{f}^{z_{f}},\left(f, z_{f}, f^{\prime}, z_{f^{\prime}}\right) \in \mathcal{R},} C_{R_{\text {sector }}}^{f} \times R_{j, f, f^{\prime}}^{z_{f}, z_{f^{\prime}}}
\end{aligned}
$$

Subject to

$$
\begin{aligned}
& \sum_{f \in \mathcal{F}: k=\operatorname{orign}_{f}} \sum_{z_{f} \in Z_{f}}\left(w_{k, t}^{f, z_{f}}-w_{k, t-1}^{f, z_{f}}\right) \leq D_{k}(t), \quad \forall k \in \mathcal{K}, \quad t \in \mathcal{T} \\
& \sum_{f \in \mathcal{F}: k=\text { dest }_{f}} \sum_{z_{f} \in Z_{f}}\left(w_{k, t}^{f, z_{f}}-w_{k, t-1}^{f, z_{f}}\right) \leq A_{k}(t) \quad \forall k \in \mathcal{K}, \quad t \in \mathcal{T} \\
& \sum_{f \in \mathcal{F}: j \in \mathcal{p}_{f}^{z_{f}: j^{\prime} \in p_{f}^{z_{f}}}} \sum_{(j+1)}\left(w_{j, t}^{f, z_{f}}-w_{j^{\prime}, t}^{f, z_{f}}\right) \leq S_{j}(t) \quad \forall j \in \mathcal{p}_{f}^{z_{f}}, \quad t \in \mathcal{T}
\end{aligned}
$$




$$
\begin{aligned}
& w_{\text {orign }_{f}, T}^{f, z_{f}}=w_{\text {dest }_{f}, T}^{f, z_{f}} \quad \forall f \in \mathcal{F}^{G} \forall, \quad z_{f} \in Z_{f} \\
& w_{j, t-1}^{f, z_{f}}-w_{j, t}^{f, z_{f}} \leq 0, \quad \forall f \in \mathcal{F}, j \in \mathcal{K} \cup p_{f}^{z_{f}}, t \in \mathcal{T}, \quad z_{f} \in Z_{f} \\
& w_{j, t}^{f, z_{f}}-w_{j^{\prime}, t-l_{j j^{\prime}}}^{f, z_{f}} \leq 0, \quad \forall f \in \mathcal{F} t \in T_{j}^{f}, j \in p_{f}^{z_{f}} \cup \operatorname{dest}_{f}: j \neq \operatorname{orign}_{f}: j^{\prime}=p_{f}^{z_{f}}(j-1), z_{f} \\
& \in Z_{f} \\
& \sum_{z_{f} \in z_{f}} w_{k, T}^{f, z_{f}}=1 \quad \forall f \in \mathcal{F}: k=\text { orign }_{f} \\
& w_{\text {orign }_{f}, t}^{f, z_{f}}-\sum_{z_{f^{\prime}} \in Z_{f^{\prime}}} w_{k, t-s_{f}}^{f^{\prime}, z_{f^{\prime}}} \leq 0 \quad \forall\left(f, f^{\prime}\right) \in \mathcal{C}, \quad t \in T_{\text {orign }}^{f, z_{f}}, z_{f} \in Z_{f} \\
& w_{j, t}^{f^{\prime}, z_{f^{\prime}}}-w_{j, t}^{f, z_{f}}-\left(1-w_{j, T}^{f, z_{f}}\right) \leq R_{f, f^{\prime}, j}^{z_{f}, z_{f}^{\prime}} \\
& \forall \forall j \in p_{f}^{z_{f}} \cup \mathcal{K},\left(f, z_{f}, f^{\prime}, z_{f^{\prime}}\right) \in \mathcal{R}, t \in T_{j}^{f, f^{\prime}, z_{f}, z_{f^{\prime}}} \\
& w_{j, t}^{f, z_{f}}-w_{j, t}^{f^{\prime}, z_{f^{\prime}}} \leq 1-R_{f, f^{\prime}, j}^{z_{f}, z_{f}^{\prime}}, \quad \forall \forall j \in p_{f}^{z_{f}} \cup \mathcal{K},\left(f, z_{f}, f^{\prime}, z_{f^{\prime}}\right) \in \mathcal{R}, t \in T_{j}^{f, f^{\prime}, z_{f}, z_{f^{\prime}}} \\
& w_{j, t}^{f, z_{f}}, R_{f, f^{\prime}, j}^{z_{f}, z_{f}^{\prime}} \in\{0,1\}
\end{aligned}
$$

The objective function (1) minimizes the total cost that consists of the airborne delay cost, the ground delay cost, the rerouting penalty cost, and the sector and destination airport reversal penalty costs, where the air and the ground delay cost coefficients are calculated using (13) and (14) respectively. The use of slight super linear coefficients $(1+\varepsilon)$, where $\varepsilon$ is a small fraction close to zero, is to ensure the fairness between the flights by providing a better distribution of the delay instead of assigning a huge amount of delay to few flights. Eq. (13) calculates the amount of arrival delay, which corresponds to the air delay after the removal of any ground delay as shown in (14).

$$
\begin{aligned}
& c_{\text {arr }}^{f}(t)=\mathrm{C}_{\text {air }}\left(t-a_{f}^{k, z_{f}^{s}}\right)^{1+\varepsilon} \\
& c_{\text {dep }}^{f}(t)=\left(\mathrm{C}_{\text {ground }}-\mathrm{C}_{\text {air }}\right)\left(t-d_{f}^{k, z_{f}}\right)^{1+\varepsilon}
\end{aligned}
$$


Constraints (2) and (3) ensure that the number of flights that depart from an airport or arrive to an airport does not exceed the predefined capacity of departure or arrival for each period in the planning horizon. Constraint (4) ensures that each sector capacity is not exceeded. Constraint (5) ensures that if a flight departs from its origin airport, it must arrive to its destination airport. Equation (6) is the time connectivity constraint, where if $w_{j, t}^{f, z_{f}}=1$ for a period, t, then it should be one for all the later periods. Constraint (7) represents the sector connectivity, where a flight cannot enter the next sector unless it has spent at least the minimum required time in its previous sectorj' ${ }^{\prime} p_{f}^{z_{f}}(j-1)$. Equation (8) limits the number of selected paths for a flight to one; modifying this constraint to at most one path allows the flight to be cancelled.

Constraint (9) connects the continuing flights, so that if a flight is continuing, then it cannot depart for its next flight unless it has spent at least its specified turnaround duration. Constraints (10) and (11) are used to count if reversal between flights at a resource $j$ occurs or not according to the cases in Table I. Reversal can occur if and only if any two flights have common resource, sector or destination airport, and a common time to enter the common resource. A flight $f^{\prime}$ is reversible if its minimum flight time is between $\overline{\bar{T}}_{j}^{f, z_{f}}<\overline{\bar{T}}_{j}^{f^{\prime}, z_{f^{\prime}}} \leq \underline{T}_{j}^{f, z_{f}}$ and the maximum time units flight $f^{\prime}$ can overtake flight $f$ is $\overline{\bar{T}}_{j}^{f^{\prime}, z_{f^{\prime}}}-\underline{T}_{j}^{f, z_{f}}-1$. Thus, the reversal period is defined by $T_{j}^{f, f^{\prime}, z_{f}, z_{f^{\prime}}}=\overline{\bar{T}}_{j}^{f^{\prime}, z_{f^{\prime}}}, \ldots, \underline{T}_{j}^{f, z_{f}}-1$.

TABLE I. POSSIBLE CASES For Flight $f$ AND $f^{\prime}$

\begin{tabular}{|c|c|c|c|c|}
\hline Case & $w_{j, T}^{f, z_{f}}$ & $w_{j, t}^{f, z_{f}}$ & $\begin{array}{r}f^{\prime}, z_{f^{\prime}} \\
w_{j, t}\end{array}$ & $R_{f, f^{\prime}, j}^{z_{f}, z_{f}^{\prime}}$ \\
\hline Case 1 & 1 & 1 & 0 & 0, Eq. (11) \\
\hline Case 2 & 1 & 0 & 1 & 1, Eq. (10) \\
\hline Case 3 & 0 & 0 & 1 & 0, Eq. (10) \\
\hline
\end{tabular}

According to Table I, assume that flight $f$ is scheduled to arrive to resource $j$ before flight $f^{\prime}$ and there is a common time period between the two flights at resource $j$, and let us assume that flight $f$ uses $z_{f}=1$ and flight $f^{\prime}$ uses $z_{f^{\prime}}=2$, then if $w_{j, T}^{f, 1}=$ 1 , and $f$ arrives before $f^{\prime}$, then there is no reversal, which is guaranteed by Constraint (11). In the second case, if flight $f^{\prime}$ arrives before flight $f$ to the resource $j$ then a reversal occurs $R_{f, f^{\prime}, j}^{1,2}=1$. However, if flight $f$ uses another path, say $z_{f}=3$, then even if flight $f^{\prime}$ arrives before flight $f$, the reversal on path $z_{f}=1$ will not be counted as flight $f$ is not using this path. In other words, 
the use of $\left(1-w_{j, T}^{f, z_{f}}\right)$ in (10) is to count the reversal if and only if the selected paths are used. Constraint (12) defines the decision variables as binary.

\section{RESUlTS AND ANALYSIS}

In this section, we show the effectiveness of the proposed approach using a simple numerical example. Consider a small network of 3 airports, 12 sectors and 10 scheduled flights. Each resource in the system has a capacity of one in each period, (i.e. sector capacity $=$ airport departure capacity $=$ airport arrival capacity $=1)$. All the flights are scheduled to depart at the same time except flight 6 , which is a continuing flight with a turnaround time of at least 3 time periods (45 minutes) after the arrival of flight 2. Each flight is required to spend at least one period in each sector. The origin and destination airports for each flight are provided in Table II. The different routes connecting between any two airports are illustrated in Fig. 1, where the solid, dashed and dotted lines represent the planned route $(\mathrm{z}=1)$, the alternative route $\left(z_{f}=2\right)$ and the alternative route $\left(z_{f}=3\right)$ respectively.

TABLE II. DEPARTURE AND ARRIVAL AIRPORTS FOR EACH FLIGHT

\begin{tabular}{|c|c|c|c|c|c|c|c|c|c|c|}
\hline Flight Number & $\mathbf{1}$ & $\mathbf{2}$ & $\mathbf{3}$ & $\mathbf{4}$ & $\mathbf{5}$ & $\mathbf{6}$ & $\mathbf{7}$ & $\mathbf{8}$ & $\mathbf{9}$ & $\mathbf{1 0}$ \\
\hline Departure Airport & 1 & 1 & 2 & 2 & 3 & 3 & 1 & 1 & 1 & 1 \\
\hline Arrival Airport & 2 & 3 & 3 & 1 & 2 & 1 & 2 & 2 & 3 & 3 \\
\hline
\end{tabular}

We use this numerical example with two models: the model proposed in this paper, Fairness-ATFMRP, and the reversal model presented in [3], i.e. Fairness-ATFM. Both models are coded in MATLAB 2014a and solved using CPLEX engine 12.6.2. We compare the characteristics of the two obtained solutions with the following costs: $C_{R}^{f}$ airport $=C_{R_{\text {sector }}}^{f}=10, C_{\text {reroute }}^{f, z_{f}}=$ $10, \mathrm{C}_{\text {air }}=150, \mathrm{C}_{\text {ground }}=50$ with $\varepsilon=0.01$. The total cost that resulted from solving the Fairness-ATFMRP is 950.858 compared to a total cost of 991.264 from the Fairness-ATFM. It is worth mentioning that both models resulted in a reversal of one unit. Table II and Table III illustrate the optimal schedule compared with the planned scheduled for the Fairness-ATFMRP model and the Fairness-ATFM model respectively. The Fairness-ATFMRP model appears to result in less maximum delay (delay of 5 units for flight 8) compared to the Fairness-ATFM (delay of 6 units for flight 1). In addition, the number of delayed flights per unit time is less in the Fairness-ATFMRP model as shown in Fig. 2.

TABLE III. GROUND AND AIR DELAY USING THE FAIRNESS-ATFMRP MODEL 


\begin{tabular}{|c|c|c|c|c|c|c|c|c|c|c|}
\hline Flight Number & 1 & 2 & 3 & 4 & 5 & 6 & 7 & 8 & 9 & 10 \\
\hline \multicolumn{11}{|c|}{ Departure } \\
\hline Scheduled & 2 & 2 & 2 & 2 & 2 & 11 & 2 & 2 & 2 & 2 \\
\hline Optimal & 5 & 2 & 2 & 5 & 2 & 11 & 3 & 7 & 6 & 4 \\
\hline (Ground delay) & (3) & $(0)$ & $(0)$ & (3) & $(0)$ & $(0)$ & (1) & $(5)$ & (4) & $(2)$ \\
\hline \multicolumn{11}{|c|}{ Arrival } \\
\hline Scheduled & 7 & 8 & 7 & 7 & 6 & 17 & 7 & 7 & 8 & 8 \\
\hline Optimal & 10 & 8 & 7 & 10 & 6 & 17 & 8 & 12 & 12 & 10 \\
\hline (Air delay) & $(0)$ & $(0)$ & $(0)$ & $(0)$ & $(0)$ & $(0)$ & $(0)$ & $(0)$ & $(0)$ & $(0)$ \\
\hline \multicolumn{11}{|c|}{ Route } \\
\hline Scheduled & 1 & 1 & 1 & 1 & 1 & 1 & 1 & 1 & 1 & 1 \\
\hline Optimal & 1 & 1 & 2 & 3 & 1 & 1 & 1 & 2 & 1 & 1 \\
\hline
\end{tabular}

TABLE IV. GROUND AND AIR DELAY USING THE FAIRNESS-ATFM MODEL

\begin{tabular}{|c|l|l|l|l|l|l|l|l|l|l|l|}
\hline $\begin{array}{c}\text { Flight } \\
\text { Number }\end{array}$ & $\mathbf{1}$ & $\mathbf{2}$ & $\mathbf{3}$ & $\mathbf{4}$ & $\mathbf{5}$ & $\mathbf{6}$ & $\mathbf{7}$ & $\mathbf{8}$ & $\mathbf{9}$ & $\mathbf{1 0}$ \\
\hline $\begin{array}{c}\text { Scheduled } \\
\text { Optimal }\end{array}$ & 2 & 2 & 2 & 2 & 2 & 11 & 2 & 2 & 2 & 2 \\
(Ground & 8 & 2 & 2 & 4 & 3 & 11 & 6 & 4 & 5 & 3 \\
delay) & $(6)$ & $(0)$ & $(0)$ & $(2)$ & $(1)$ & $(0)$ & $(4)$ & $(2)$ & $(3)$ & $(1)$ \\
\hline
\end{tabular}


(i)

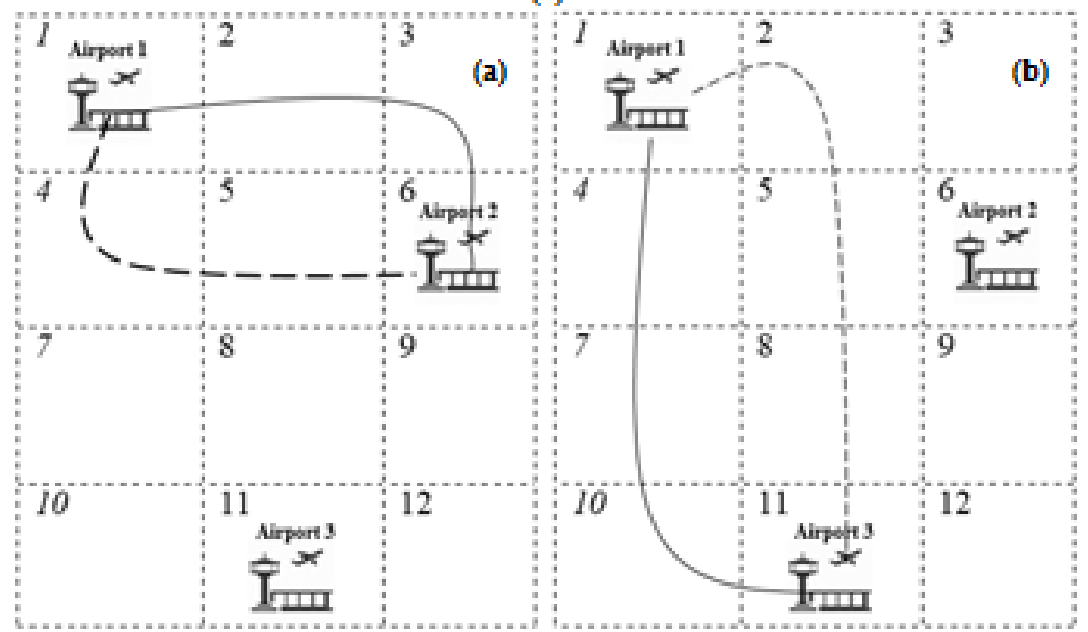

(ii)
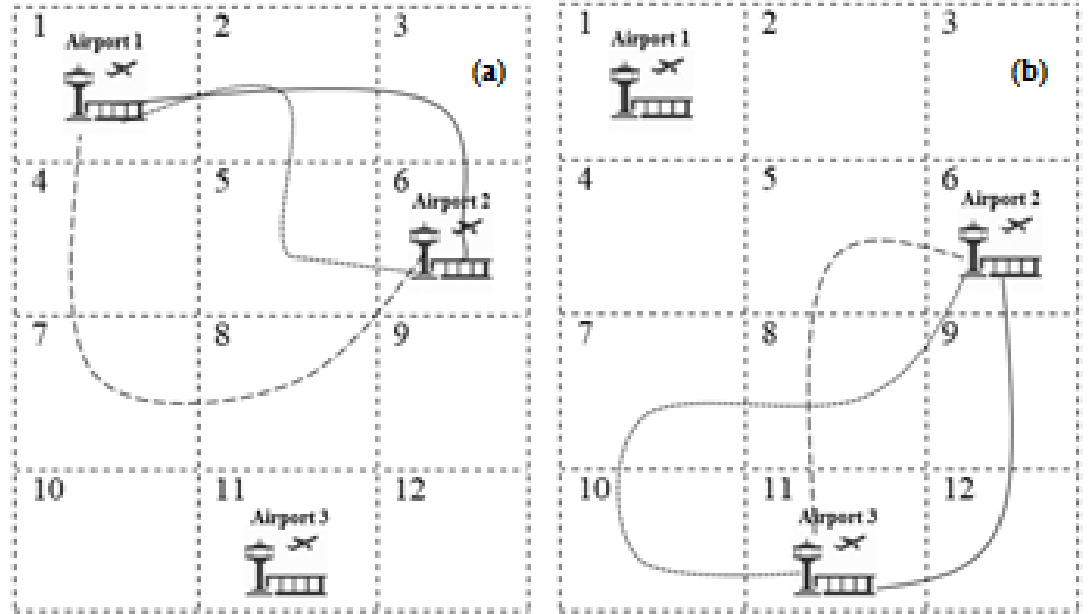

(iii)
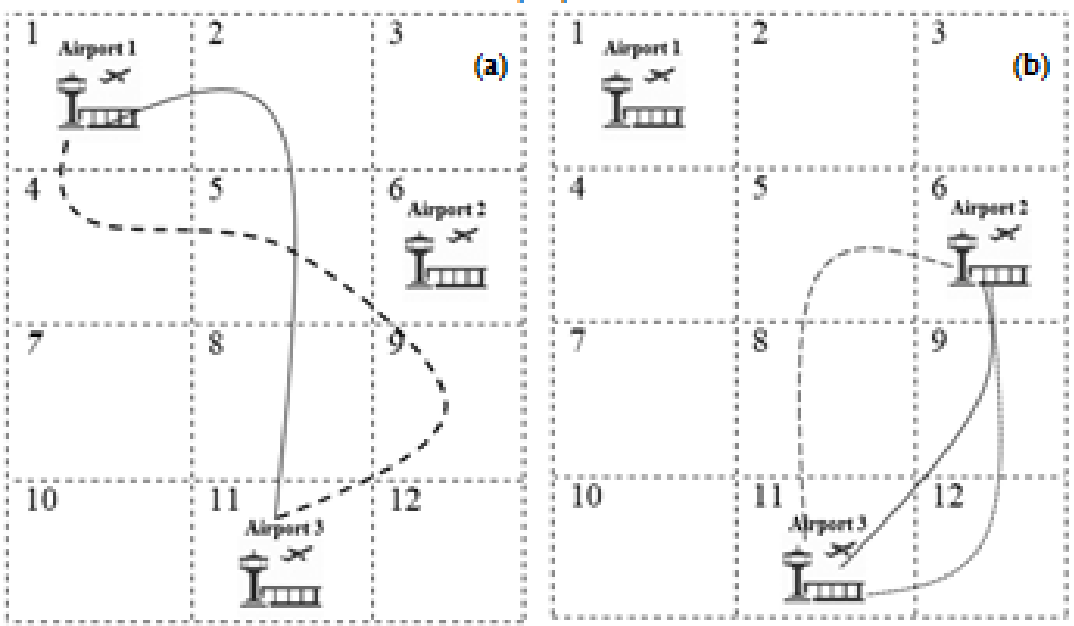

Figure 1. Different routes for flights departing (i) from airport 1 to (a) airport 2 (b) airport 3, (ii) from airport 2 to (a) airport 1 (b) airport 3, and (iii) from airport 3 to (a) airport 1 (b) airport 2, 


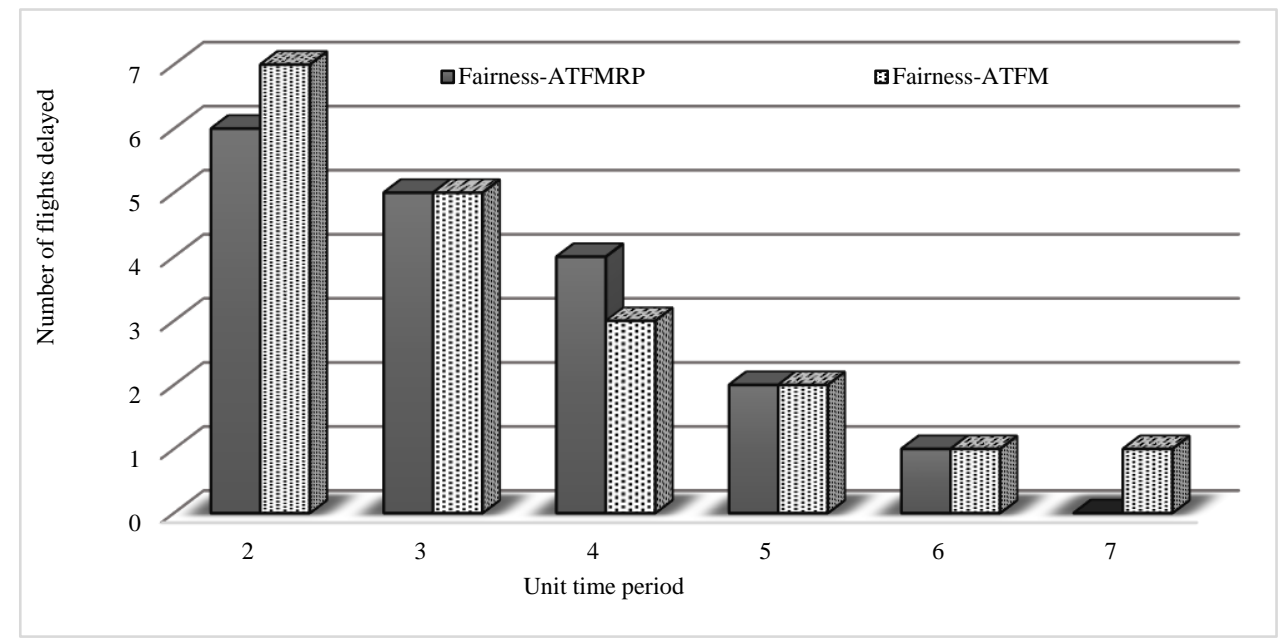

Figure 2. Number of delay flights per time unit (Fairness-ATFMRP vs. Fairness-ATFM)

\section{CONCLUSION}

In this paper, we extended the Fairness-ATFM model presented in [3] to account for rerouting in order to better utilize the airspace and to reduce the congestion. We proposed therefore a Fairness-ATFMRP model that resulted in less delay and less number of delayed flights compared to the model without rerouting. Generally, according to Bertsimas and Gupta [3], the computation difficulties and the lack of acceptable fairness concepts makes network models unpractical. As a future work, there is a need to have an overall enhanced fairness concept and to focus more on the practicality of the developed models.

\section{ACKNOWLEDGMENT}

The authors gratefully acknowledge the support received from the University of Sharjah, Emirates Islamic Bank, and CentraleSupélec.

\section{REFERENCES}

[1] A. Mukherjee and M. Hansen, "A dynamic rerouting model for air traffic flow management," Transp. Res. Part B Methodol., vol. 43, no. 1, pp. 159-171, 2009. 
[2] A. Agustín, A. Alonso-Ayuso, L. F. Escudero, and C. Pizarro, "On air traffic flow management with rerouting. Part I: Deterministic case,” Eur. J. Oper. Res., vol. 219, no. 1, pp. 156-166, 2012.

[3] D. Bertsimas and S. Gupta, "Fairness and Collaboration in Network Air Traffic Flow Management: An Optimization Approach,” Transp. Sci., vol. 50, no. 1, pp. 57-76, 2015.

[4] D. Bertsimas and S. S. Patterson, "The air traffic flow management problem with enroute capacities," Oper. Res., vol. 46, no. 3, pp. 406-422, 1998.

[5] O. Richetta and A. Odoni, "Solving optimally the static ground-holding policy problem in air traffic control," Transportation Science, vol. 27, no. 3. pp. 228-238, 1993.

[6] O. Richetta and A. R. Odoni, "Dynamic solution to the ground-holding problem in air traffic control," Transp. Res. Part A, vol. 28, no. 3, pp. 167-185, 1994.

[7] G. Andreatta and L. Brunetta, "Multiairport Ground Holding Problem: A Computational Evaluation of Exact Algorithms," Oper. Res., vol. 46, no. 1, pp. 57-64, 1998.

[8] J. Chen, L. Chen, and D. Sun, "Air traffic flow management under uncertainty using chance-constrained optimization," Transp. Res. Part B Methodol., vol. 102, pp. 124-141, 2017.

[9] D. J. Bertsimas, G. Lulli, and A. Odoni, "The air traffic flow management problem: an integer optimization approach," 3th Int. Conf. Integer Program. Comb. Optim., pp. 34-46, 2008.

[10] S. Gupta and D. Bertsimas., "Multistage Air Traffic Flow Management under Capacity Uncertainty: A Robust and Adaptive Optimization Approach,” in 51st AGIFORS Annual Proceedings: annual symposium and study group meeting, 2011, pp. 692-721.

[11] A. Alonso, L. F. Escudero, and M. T. Ortuno, "A stochastic 0-1 program based approach for the air traffic flow management problem,” Eur. J. Oper. Res., vol. 120, no. 1, pp. 47-62, 2000.

[12] G. Andreatta, P. Dell 'olmo, and G. Lulli, "An aggregate stochastic programming model for air traffic flow management," Eur. J. Oper. Res., vol. 215, no. 3, pp. 697-704, 2011.

[13] D. Bertsimas and S. S. Patterson, "The Traffic Flow Management Rerouting Problem in Air Traffic Control: A Dynamic Network Flow Approach,” Transp. Sci., vol. 34, no. 3, pp. 239-255, 2000. 
[14] D. Bertsimas, G. Lulli, and A. Odoni, “An integer optimization approach to large-scale air traffic flow management,” Oper. Res., vol. 59, no. 1, pp. 211-227, 2011.

[15] M. O. Ball, R. Hoffman, A. R. Odoni, and R. Rifkin, “A Stochastic Integer Program with Dual Network Structure and Its Application to the Ground-Holding Problem," Oper. Res., vol. 51, no. March 2015, pp. 167-171, 2003.

[16] A. Mukherjee and M. Hansen, "Dynamic stochastic optimization model for air traffic flow management with en route and airport capacity constraints," in 6th USA/Eur. Air Traffic Manage. R\&D Seminar, 2005.

[17] G. Lulli and A. Odoni, “The European Air Traffic Flow Management Problem,” Transp. Sci., vol. 41, no. 4, pp. 431-443, 2007.

[18] A. Agustín, A. Alonso-Ayuso, L. F. Escudero, and C. Pizarro, “On air traffic flow management with rerouting. Part II: Stochastic case,” Eur. J. Oper. Res., vol. 219, no. 1, pp. 167-177, 2012. 